Ich danke den Autorinnen und Autoren für ihr grosses Engagement und die fristgerechte Lieferung der Beiträge. Frau Dipl.-Kffr. Daniela B. Schäfer (Lehrstuhl für Marketing und Unternehmensführung, Universität Basel) sei zudem herzlich für ihre starke operative Unterstützung während der ganzen Entstehungsphase des Themenheftes gedankt. Ich wünsche Ihnen eine anregende Lektüre.

Ihr Manfred Brubn

\title{
Best Paper Award
}

Der Beitrag „Risk Management from an Organizational Psychology Perspective: A Decision Process for Managing Uncertainties“ von Gudela Grote (Heft 1/2011) verdient aus drei primären Gründen den Best Paper Award 2011 von „Die Unternehmung“: Er behandelt erstens ein relevantes Thema von allgemeiner Bedeutung im Risikomanagement, trägt dazu zweitens eine Vielzahl an innovativen Inhalten bei und schlägt drittens eine Brücke zwischen Theorie und Praxis.

Das Thema ist relevant, weil es um eine Hauptaufgabe des Risikomanagements geht: das Management von Unsicherheit. Unsicherheit wird als „neutrale“ Quelle von Risiko verstanden. Ein angemessener Umgang mit Unsicherheit ist somit eine wesentliche Voraussetzung für ein effektives Risikomanagement. Die wissenschaftliche Literatur zum Risikomanagement konzentriert sich überwiegend auf die Identifikation und Bewertung des Risikos und weniger auf die Entscheidungsfindung in Abhängigkeit verschiedener Strategien des Unsicherheitsmanagements. Insbesondere die Diskussion über die Strategie der Unsicherheitserhöhung, die beispielsweise im Zuge der Bildung neuer Geschäftsmöglichkeiten im Finanzsektor relevant ist, stand bislang nicht im Fokus wissenschaftlicher Arbeiten.

Der Beitrag ist innovativ und zwar in mehrfacher Hinsicht. Die Autorin zieht die Reduktion, den Erhalt sowie die Erhöhung von Unsicherheit als potenziell wirkungsvolle Strategien ein, die bisher in Abhandlungen über Unsicherheit und Risiko vernachlässigt wurden. Sie schlägt zudem einen Entscheidungsprozess vor, der die Entscheidungsträger bei der Wahl der genannten Strategien unterstützt und sieht hierbei als übergeordnetes Ziel die Balance zwischen Stabilität und Flexibilität der Geschäftsprozesse an. Der Beitrag nimmt eine organisationspsychologische Perspektive ein und leitet schliesslich Schlussfolgerungen zur Rolle des Risikomanagements in Organisationen ab.

Der Beitrag verbindet Theorie und Praxis, indem er den vorgestellten Entscheidungsprozess anhand realer Daten des Risikomanagements eines Eisenbahnunternehmens durchspielt. Als ein Brückenschlag zwischen Theorie und Praxis ist ausserdem anzusehen, dass die Autorin ihre Erkenntnisse in wissenschaftlich präziser und zugleich gut lesbarer Form präsentiert.

Prof. Dr. Manfred Brubn, Universität Basel 
Die Herausgeber der Zeitschrift «Die Unternehmung» verleihen

\author{
Prof. Dr. Gudela Grote
}

für ihren im Heft 1/2011 publizierten Beitrag «Risk Management from an

Organizational Psychology Perspective: A Decision Process for Managing Uncertainties»

den

\title{
"Best Paper Award 2011"
}

Der Award wird verliehen für Publikationen mit einer besonders hohen wissenschaftlichen Güte und Tragweite. Die Preisträgerin hat ein wichtiges Thema von allgemeiner Relevanz behandelt, Innovatives präsentiert und eine Brücke zwischen Forschung und Praxis geschlagen. Somit hat sie einen bedeutenden Beitrag zur Förderung der wissenschaftlichen Qualität und des Renommees der Zeitschrift «Die Unternehmung» geleistet. Dafür sind die Herausgeber der Preisträgerin sehr dankbar.

Zürich, im März 2012

Prof. Dr. Dieter Pfaff Geschäftsführender Herausgeber 